\title{
The Clinical and Molecular Characteristics of Sex-Determining Region Y-Box 2 and its Prognostic Value in Breast Cancer: A Systematic Meta-Analysis
}

\author{
Gang Zhao ${ }^{\mathrm{a}}$ Xiaozhen Wang ${ }^{\mathrm{a}}$ Limei Qu ${ }^{\mathrm{b}}$ Zhu Zhu ${ }^{\mathrm{a}} \quad$ Jinghui Hong ${ }^{\mathrm{a}}$ \\ Haiqin $\mathrm{Hou}^{\mathrm{a}}$ Zuonong $\mathrm{Li}^{\mathrm{a}}$ Jun Wang ${ }^{\mathrm{a}}$ Zheng $\mathrm{Iv}^{\mathrm{c}}$ \\ ${ }^{a}$ Department of Breast Surgery, the First Affiliated Hospital of Jilin University, Changchun, China; ${ }^{b}$ Department \\ of Pathology, the First Affiliated Hospital of Jilin University, Changchun, China; 'Cancer Center, the First Affiliated \\ Hospital of Jilin University, Changchun, China
}

Keywords

SOX2 - HER2 - EGFR · Prognosis · Breast cancer

\begin{abstract}
Objective: Transcription factor SOX2 (sex-determining region Y-box 2) has a crucial role in the maintenance of the stem cell state. However, current evidence regarding the role of SOX2 in breast cancer is conflicting. We conducted this meta-analysis to clarify the association of SOX2 expression with clinical and molecular features and its prognostic effect on breast cancer. Methods: All relevant articles were searched using electronic databases. The pooled odds ratios (ORs) or hazard ratios (HRs: multivariate Cox survival analysis) with their $95 \%$ confidence intervals (Cls) were calculated. Results: A final total of 18 studies containing 3,080 patients with breast cancer were included. SOX2 protein expression was not related to age, menopausal status, lymph node metastasis, lymphovascular invasion, molecular estrogen receptor status, progesterone receptor status, triple-negative status, and the overall survival in breast cancer, but was closely associated with advanced tumor grade (grade 3 vs. grade $1-2: \mathrm{OR}=2.74,95 \% \mathrm{Cl}=1.85-4.06, p<0.001)$, clinical stage (stage $3-4$ vs. stage $0-2: O R=2.46,95 \% \mathrm{Cl}=1.37-4.40$, $p=0.002$ ), pT stage (T stage $2-4$ vs. T stage $1: O R=1.52,95 \%$ $\mathrm{Cl}=1.07-2.17, p=0.019)$, molecular human epidermal
\end{abstract}

growth factor receptor 2 (HER2) status (positive vs. negative: $\mathrm{OR}=1.61,95 \% \mathrm{Cl}=1.21-2.14, p=0.001)$, epidermal growth factor receptor (EGFR) status (positive vs. negative: OR = $2.21,95 \% \mathrm{Cl}=1.13-4.33, p=0.021)$, and worse disease-free survival (DFS) $(\mathrm{HR}=2.66,95 \% \mathrm{Cl}=1.20-5.91, p=0.016)$ of breast cancer. Conclusions: SOX2 expression is correlated with breast cancer progression, HER2 status, and EGFR status, and may be an independent prognostic marker for predicting poor DFS.

(c) 2020 S. Karger AG, Basel

\section{Introduction}

Breast cancer is the most common female malignancy and the leading cause of death in female malignant tumors worldwide [1]. Based on global cancer statistics, approximately 2,088,849 new patients are clinically diagnosed with breast carcinoma, leading to approximately 626,679 deaths due to breast cancer worldwide in 2018 [1]. Breast cancer is divided into different subtypes based on clinical or molecular characteristics, including endocrine (estrogen [ER] and progesterone $[\mathrm{PR}]$ ) receptors, human epidermal growth

Gang Zhao and Xiaozhen Wang contributed equally to this paper. 
Fig. 1. Flow diagram of the literature search and study selection.

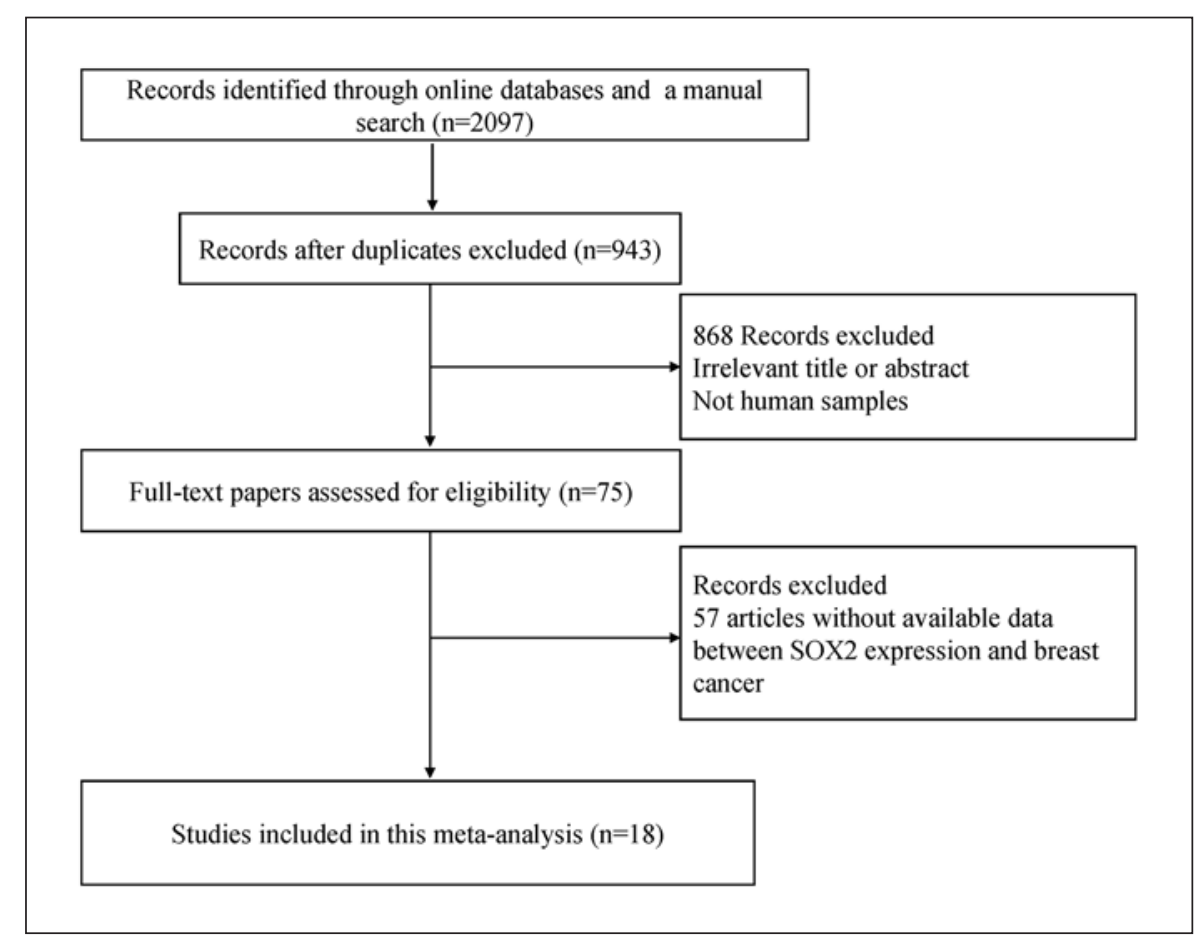

factor receptor 2 (HER2), and triple-negative (TN: a lack of ER, PR, and HER2 expression) or basal-like $[2,3]$. However, the satisfactory survival rate remains to be improved; the mechanism of the clinical survival for patients with breast cancer has yet to be fully understood $[4,5]$. Therefore, there is a pressing need to develop novel useful biomarkers to establish the optimal therapeutic strategy.

Cancer stem cells (CSCs), a subset of cells within the tumor, have the potential for self-renewal, unlimited proliferation and differentiation, and tumor formation $[6,7]$. CSCs are thought to be a major cause for the failure of cancer therapy due to their considerable resistance to conventional therapy such as chemotherapy or radiation therapy, and contribute to tumor progression, metastasis, and unfavorable prognosis [8-10]. Sex-determining region Y-box 2 (SOX2), located on human chromosome 3q26.3-q27, encodes a protein containing 317 amino acids [11]. SOX2 contains a high mobility group DNAbinding domain and is a member of the SOX family of transcriptional regulators $[11,12]$. It regulates the properties of CSC self-renewal and pluripotency and plays a critical role in the maintenance of the stem cell state [13, 14]. SOX2 is also an important regulator of embryonic development and essential for cellular reprogramming processes and has a role in tissue homeostasis and regeneration [15]. It is involved in cancer cell migration, invasion, and proliferation, cell cycle progression, metastasis formation, and apoptosis [14]. CSC marker SOX2 is frequently expressed in many human cancers, such as gastric cancer, colorectal cancer, lung cancer, and ovarian carci- noma [16]. Some studies show that SOX2 expression is associated with poor prognosis in some cancers such as esophageal, colorectal, and head and neck cancers [17, 18]. However, SOX2 expression is correlated with a favorable prognosis in non-small cell lung cancer [19]. Recent studies reported that SOX2 expression was detected in breast cancer and may be related to the worse prognosis of breast cancer [20-23].

The role of SOX2 expression remains controversial in breast cancer. For example, Gwak et al. [24] (2017) reported that SOX2 expression was associated with advanced tumor grade of breast cancer. Liu et al. [22] 2018 showed no association between SOX2 expression and tumor grade of breast cancer. Therefore, the purpose of this meta-analysis was to assess the clinical, molecular, and prognostic values of SOX2 expression in breast cancer.

\section{Materials and Methods}

\section{Literature Search}

A systematic literature search was conducted to achieve eligible studies from PubMed, EMBASE, Web of Science, and Cochrane Library databases up to July 3, 2019. The keywords and search terms were applied as follows: "SOX2 OR SOX-2 OR Sex-determining region $\mathrm{Y}$-box protein 2 OR Sex determining region $\mathrm{Y}$ box2 OR Sex-determining region Y-box 2 OR SRY box-2," "mammary OR breast," "cancer OR tumor OR neoplasm OR carcinoma." The reference list reported in the eligible studies was also screened to identify other relevant articles. This meta-analysis was applied based on the Preferred Reporting Items for Systematic Reviews and Meta-analysis (PRISMA) statement criteria [25]. 
Table 1. Main characteristics of the included studies

\begin{tabular}{|c|c|c|c|c|c|c|c|c|c|c|c|}
\hline First author & Country & $\begin{array}{l}\text { Age, } \\
\text { years }\end{array}$ & Method & Stage & Antibodies & Sources of antibodies & Staining & $\begin{array}{l}\text { Cut-off } \\
\text { values }\end{array}$ & $N(\mathrm{E}+\%)$ & $\begin{array}{l}\text { Clinical } \\
\text { features }\end{array}$ & $\begin{array}{l}\text { Survival } \\
\text { (MA) }\end{array}$ \\
\hline $\begin{array}{l}\text { Rodriguez-Pinilla } \\
{[30], 2007}\end{array}$ & Spain & NA & IHC & NA & anti-SOX2 & Stem-Cell Technologies & nuclear & any & $198(16.7)$ & yes & \\
\hline $\begin{array}{l}\text { Lengerke } \\
{[31], 2011}\end{array}$ & Germany & $50-69$ & IHC & NA & anti-SOX2 & $\begin{array}{l}\text { AF2018, R\&D Systems, } \\
\text { heat-induced epitope } \\
\text { retrieval (HIER) }\end{array}$ & nuclear & $>0 \%$ & $86(27.9)$ & yes & \\
\hline $\begin{array}{l}\text { Leis } \\
{[26], 2012}\end{array}$ & Spain & 55 & IHC & $1-3$ & NA & $\mathrm{NA}$ & nuclear & $>0 \%$ & $160(15)$ & yes & \\
\hline $\begin{array}{l}\mathrm{Li} \\
{[32], 2013}\end{array}$ & China & NA & IHC & $1-4$ & anti-SOX2 & $\begin{array}{l}\text { ab75485, Abcam Inc., } \\
\text { Cambridge, UK }\end{array}$ & NA & $\geq 30 \%$ & $269(46.5)$ & yes & \\
\hline $\begin{array}{l}\text { Nagata } \\
{[34], 2014}\end{array}$ & Japan & 55.2 & IHC & $0-3$ & anti-SOX2 & $\begin{array}{l}\text { LV1614793, Millipore } \\
\text { Corp., Billerica, } \\
\text { MA, USA }\end{array}$ & nuclear & $3-5$ scores & $100(34)$ & yes & \\
\hline $\begin{array}{l}\text { Piva } \\
\text { [35], } 2014\end{array}$ & Spain & NA & IHC & NA & anti-SOX2 & $\begin{array}{l}\text { Stem-Cell Technologies, } \\
\text { Cat No. } 01438\end{array}$ & nuclear & NA & $81(59.3)$ & yes & \\
\hline $\begin{array}{l}\text { Abd El-Maqsoud } \\
{[33], 2014}\end{array}$ & Egypt & 53.37 & IHC & NA & anti-SOX2 & $\begin{array}{l}\text { clone 57CT23.3.4, Abcam, } \\
\text { Cambridge, MA, USA }\end{array}$ & nuclear & any & $126(33.3)$ & yes & \\
\hline $\begin{array}{l}\text { Huang } \\
{[20], 2014}\end{array}$ & China & NA & IHC & NA & anti-SOX2 & cloneSP76, Ventana & nuclear & $\geq 1 \%$ & $552(19)$ & yes & DFS \\
\hline $\begin{array}{l}\text { Shima } \\
{[36], 2016}\end{array}$ & Japan & 60.5 & IHC & NA & anti-SOX2 & Abnova, Taipei, Taiwan & nuclear & any & $102(8.8)$ & yes & OS, DFS \\
\hline $\begin{array}{l}\text { Gwak } \\
{[24], 2017}\end{array}$ & Korea & 50.9 & IHC & NA & anti-SOX2 & $\begin{array}{l}\text { rabbit monoclonal, clone SP76, } \\
\text { Ventana }\end{array}$ & nuclear & $\geq 1 \%$ & $319(10.3)$ & yes & \\
\hline $\begin{array}{l}\text { Wang } \\
{[38], 2017}\end{array}$ & China & NA & IHC & $1-4$ & anti-SOX2 & Cell Signaling Technology & nuclear & NA & $74(25.7)$ & yes & \\
\hline $\begin{array}{l}\mathrm{Ni} \\
{[37], 2017}\end{array}$ & China & NA & IHC & $1-4$ & anti-SOX2 & $\begin{array}{l}\text { R\&D Systems, } \\
\text { Minneapolis, MN, USA }\end{array}$ & NA & 2 or 3 scores & $127(33.1)$ & yes & OS \\
\hline $\begin{array}{l}\text { Gupta } \\
\text { [39], } 2018\end{array}$ & Canada & NA & IHC & NA & $\begin{array}{l}\text { anti- } \\
\text { pSOX2T116 }\end{array}$ & $4 \mathrm{C} 7 \mathrm{G} 2$ & nuclear & $\begin{array}{l}\text { an average score of } 20 \\
\text { in the } 5 \text { fields }\end{array}$ & $35(37.1)$ & yes & \\
\hline $\begin{array}{l}\text { Zhang } \\
\text { [41], 2018 }\end{array}$ & China & 54.35 & $\mathrm{IHC} / \mathrm{IF}$ & $1-3$ & anti-SOX2 & CST, L1D6A2, USA & $\begin{array}{l}\text { cell } \\
\text { membrane }\end{array}$ & $\geq$ HSCORE 0.7 & $127(16.5)$ & yes & \\
\hline $\begin{array}{l}\mathrm{Li} \\
{[40], 2018}\end{array}$ & China & NA & IHC & NA & anti-SOX2 & ab97959, Abcam & NA & $\geq 1$ score & $70(72.9)$ & yes & \\
\hline $\begin{array}{l}\text { Kündig } \\
{[21], 2018}\end{array}$ & Switzerland & NA & $\mathrm{IHC}$ & NA & anti-SOX2 & cloneEPR3131, Ventana & $\begin{array}{l}\text { cytoplasm/ } \\
\text { nuclear }\end{array}$ & $>0 \%$ & $283(9.5)$ & yes & \\
\hline $\begin{array}{l}\text { Liu } \\
{[22], 2018}\end{array}$ & China & 50.93 & IHC & $1-4$ & anti-SOX2 & Santa Cruz, CA, USA & NA & $\geq 2$ scores & $237(51.5)$ & yes & \\
\hline $\begin{array}{l}\text { Yang } \\
{[23], 2018}\end{array}$ & China & 53.8 & IF & $1-3$ & anti-SOX2 & CST & NA & HScore $\geq 0.7$ & $134(20.9)$ & yes & OS \\
\hline
\end{tabular}

NA, not applicable; IHC, immunohistochemistry; IF, immunofluorescence; MA, multivariate Cox analysis; OS, overall survival; DFS, disease-free survival; $N$, the number of the study population; E+, positive expression; HSCORE, histological score.

\section{Eligibility Criteria}

The published studies for inclusion in this meta-analysis were required to meet the following criteria: (1) the diagnosis of patients with breast cancer was made by pathological examination; (2) the expression status of SOX2 protein in tissue samples was detected based on immunohistochemistry or immunofluorescence; (3) SOX2 was defined as "positive" or "negative" from the eligible studies; (4) studies provided available data to assess the correlation of SOX2 expression with clinical and molecular features; (5) studies reported the values of hazard ratios (HRs) and 95\% confidence intervals (CIs) to evaluate the association between SOX2 expression and survival status using multivariate Cox analysis; and (6) the corresponding author was contacted via email and requested to supply sufficient data when essential data were unavailable from the original article. Only the most complete paper or the most recently published paper was included when authors published multiple articles using the overlapping sample data. The main exclusion criteria were: (1) abstracts, letters, review articles, or case reports; (2) cell lines, animal studies, or blood samples; and (3) studies without sufficient data.

\section{Data Extraction}

Data extraction was conducted from the included studies, including age, menopausal status, tumor grade, clinical stage, pT stage, lymph node metastasis, lymphovascular invasion, ER status, PR status, HER2 status, TN status, and epidermal growth factor receptor (EGFR) status, and survival data (HRs and their 95\% CIs) using multivariate Cox analysis. In the case of data regarding TN status, breast cancer with basal-like tumor type was considered as TN based on the references $[20,26]$.

\section{Statistical Analysis}

All data analyses were conducted using version 12.0 Stata statistical software (Stata Corp, College Station, TX, USA). The pooled odds ratios (ORs) and 95\% CIs were used to estimate the strength of the association between SOX2 expression and clinical and molecular features of breast cancer, including age, menopausal status, tumor grade, clinical stage, $\mathrm{pT}$ stage, lymph node metastasis, lymphovascular invasion, ER status, PR status, HER2 status, TN status, and EGFR status. The pooled HRs and their 95\% CIs were used to assess the survival significance of SOX2 expression on breast cancer 


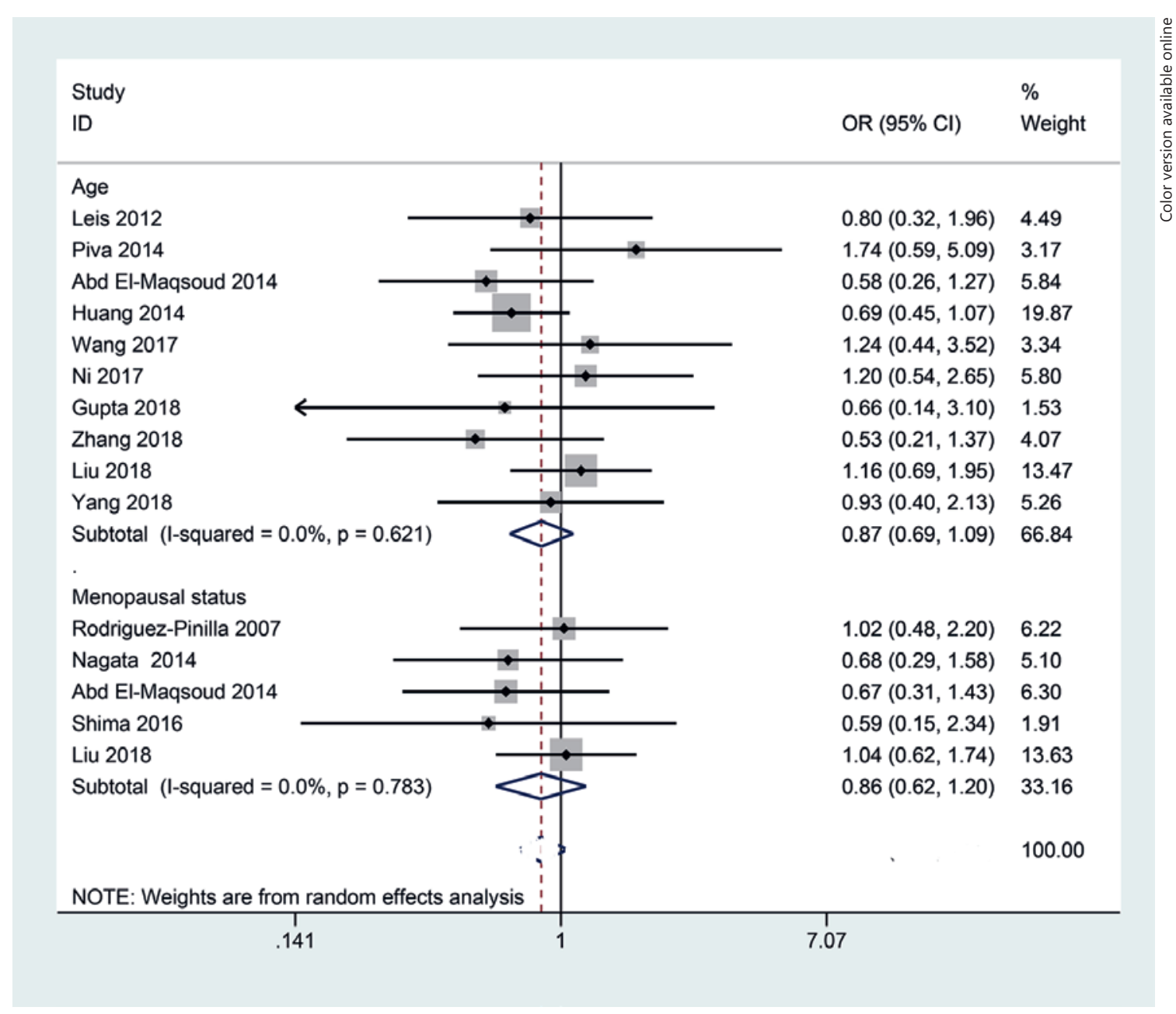

Fig. 2. Forest plot of the association between SOX 2 expression and age ( $\geq 50$ vs. $\leq 50$ years) and menopausal status (postmenopausal vs. premenopausal).

patients. The random-effects model (DerSimonian-Laird method) was used in the present meta-analysis. The assessment of statistical heterogeneity was measured based on the Cochran Q statistic [27]. A $p$ value of $Q$ test $<0.1$ was considered as significant heterogeneity. When substantial heterogeneity was measured, sensitivity analyses were performed to determine the influence of single study and stability of the re-calculated results by omitting an individual study [28]. Evidence of publication bias was detected using Egger's test for the results with more than 8 studies, $p<0.05$ was considered to be statistically significant publication bias [29].

\section{Results}

\section{Characteristics of the Eligible Studies}

The detailed steps of the literature search and selection procedures of the included studies are shown in Figure 1. According to the selection criteria, the final 18 studies [20-24, 26, 30-41] were included in this meta-analysis, including 3,080 patients with breast cancer. The eligible studies were published from 2007 to 2018, which were conducted in China, Japan, Korea, Canada, Egypt, Spain,
Switzerland, and Germany. Of these eligible studies, all studies evaluated the relationships of SOX2 expression and clinical and molecular features of breast cancer. Four studies estimated the survival of SOX2 expression on breast cancer prognosis based on multivariate Cox analysis $[20,23,36,37]$. Table 1 and online supplementary Table S1 (for all online suppl. material, see www.karger. com/doi/10.1159/000505806) summarize the main characteristics of the eligible studies.

\section{Correlation between SOX2 Expression and Clinical Features of Breast Cancer}

No association was found between SOX2 expression and age ( $n=10$ studies with 1,645 patients; $\geq 50$ vs. $\leq 50$ years: $\mathrm{OR}=0.87,95 \% \mathrm{CI}=0.69-1.09, p=0.23)$ and menopausal status ( $n=5$ studies with 759 patients; postmenopausal vs. premenopausal: $\mathrm{OR}=0.86,95 \% \mathrm{CI}=0.62-1.20$, $p=0.383$ ) (Fig. 2). A total of 17 studies with 2,862 breast cancer cases assessed the association between SOX2 expression and tumor grade, which showed that SOX2 expression was significantly correlated with advanced tu- 


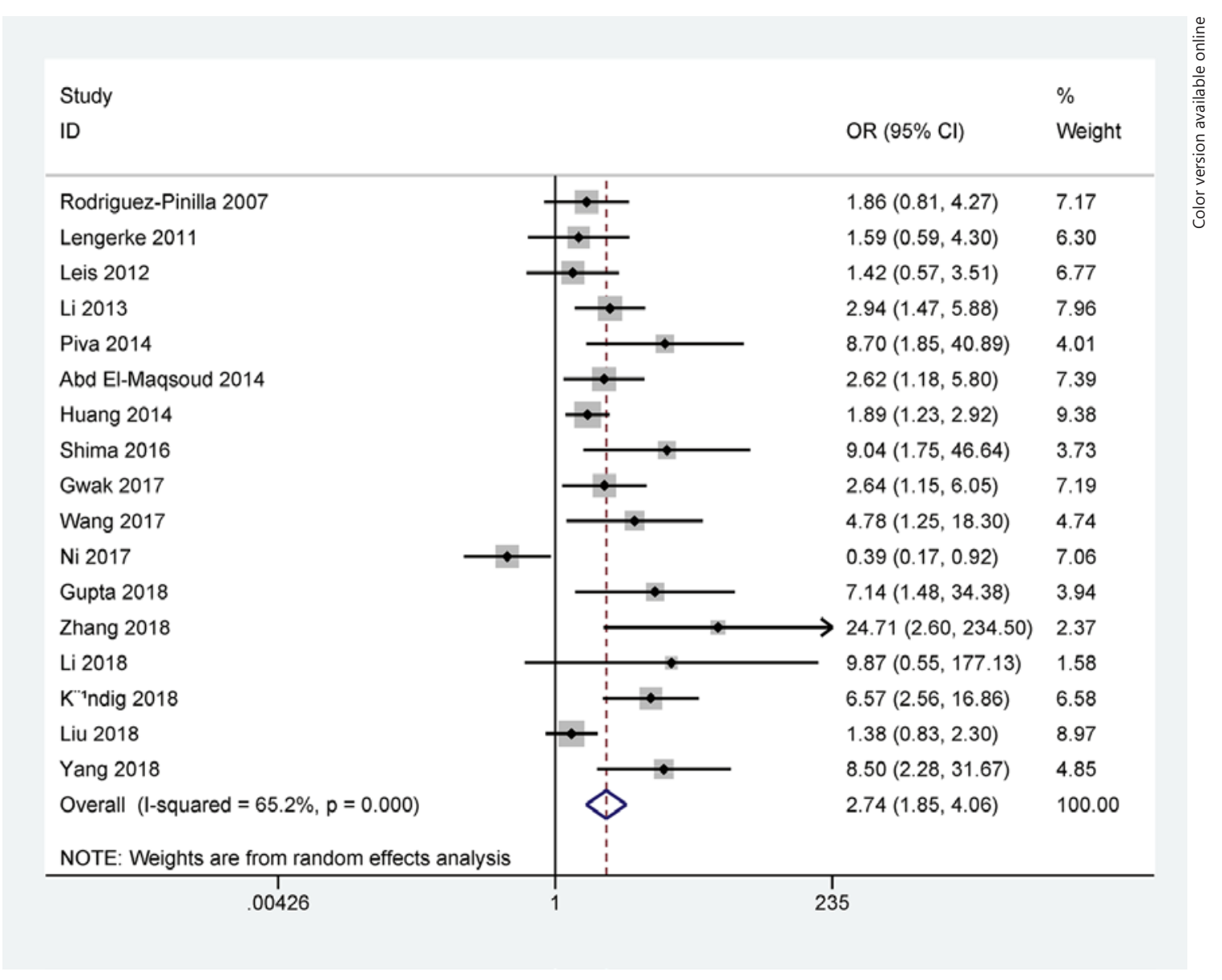

Fig. 3. Forest plot of the association between SOX 2 expression and tumor grade (grade 3 vs. grade 1-2).

mor grade (grade 3 vs. grade $1-2: \mathrm{OR}=2.74,95 \% \mathrm{CI}=$ 1.85-4.06, $p<0.001$ ) (Fig. 3).

SOX2 expression was significantly associated with advanced clinical stage (stage $3-4$ vs. stage $0-2$ : $\mathrm{OR}=2.46$, $95 \% \mathrm{CI}=1.37-4.40, p=0.002)$ and $\mathrm{pT}$ stage (T stage $2-4$ vs. $\mathrm{T}$ stage $1: \mathrm{OR}=1.52,95 \% \mathrm{CI}=1.07-2.17, p=0.019)$ (Fig. 4), including 7 studies with 1,095 patients concerning clinical stage and 13 studies with 2,041 patients concerning pT stage. No relationship was observed between SOX2 expression and lymph node metastasis $(n=12$ studies with 2,069 cases; positive vs. negative: $\mathrm{OR}=1.85$, $95 \% \mathrm{CI}=0.93-3.66, p=0.078)$ and lymphovascular invasion ( $n=5$ studies with 836 cases; positive vs. negative: $\mathrm{OR}=1.98,95 \% \mathrm{CI}=0.98-4.01, p=0.057$ ) (Fig. 5).

Correlation between SOX2 Expression and Molecular Features of Breast Cancer

No relationship was found between SOX2 expression and ER status (12 studies with 2,010 patients; positive vs. negative: $\mathrm{OR}=1.05,95 \% \mathrm{CI}=0.62-1.77, p=0.867)$ and PR status (10 studies with 1,831 patients; positive vs. negative: $\mathrm{OR}=0.98,95 \% \mathrm{CI}=0.63-1.52, p=0.923$ ) (Fig. 6). Data from 10 studies with 1,816 cases showed that SOX2 expression was significantly correlated with HER2 status (positive vs. negative: $\mathrm{OR}=1.61,95 \% \mathrm{CI}=1.21-2.14, p=$ 0.001) (Fig. 7).

No correlation was observed between SOX2 expression and TN status (yes vs. no: $\mathrm{OR}=1.70,95 \% \mathrm{CI}=0.84$ 3.45, $p=0.138$ ) (Fig. 7), including 9 studies with 1,658 cases. Data from 3 studies with 799 cases demonstrated that SOX2 expression was significantly associated with EGFR status (positive vs. negative: $\mathrm{OR}=2.21,95 \% \mathrm{CI}=$ $1.13-4.33, p=0.021$ ) (Fig. 7).

\section{Prognostic Role of SOX2 Expression Using}

Multivariate Cox Survival Analysis

SOX2 expression was not associated with overall survival $(\mathrm{OS})(\mathrm{HR}=0.90,95 \% \mathrm{CI}=0.30-2.74, p=0.859)$ in 3 studies with 363 patients, but was correlated with worse disease-free survival (DFS) $(\mathrm{HR}=2.66,95 \% \mathrm{CI}=1.20$ $5.91, p=0.016$ ) in 2 studies with 205 patients (Fig. 8).

\section{Heterogeneity Analyses}

Heterogeneity was observed between SOX2 expression and tumor grade, clinical stage, pT stage, lymph node metastasis, lymphovascular invasion, ER status, PR 


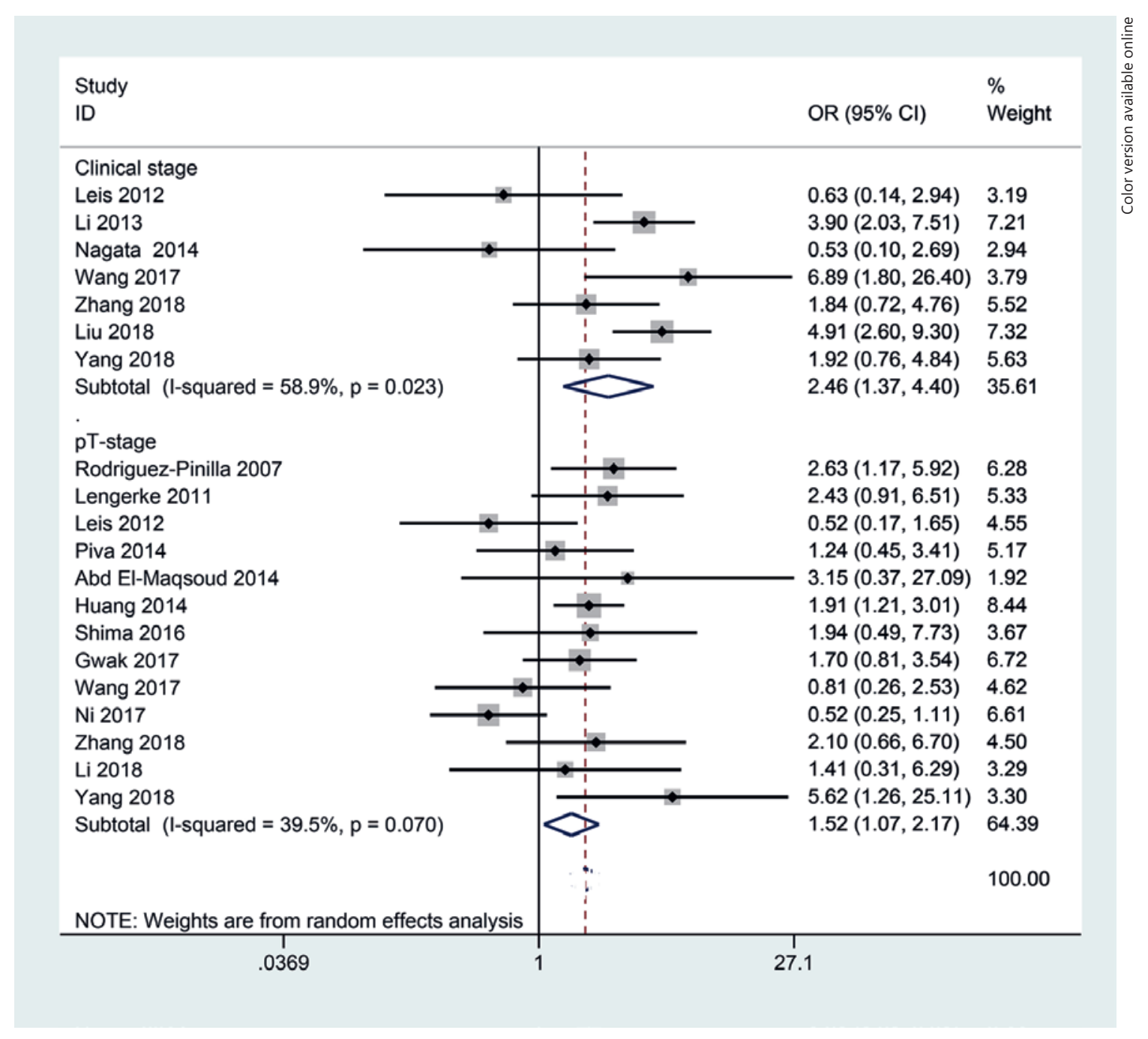

Fig. 4. Forest plot of the association between SOX 2 expression and clinical stage (stage 3-4 vs. stage $0-2$ ) and pT stage (T stage $2-4$ vs. T stage 1 ).

status, and TN status (all $p<0.1$ ). Sensitivity analyses were conducted in this meta-analysis. When 3 studies $[20,22,37]$ were removed, the re-calculated OR remained significant between SOX2 expression and tumor grade $(\mathrm{OR}=$ $3.49, p<0.001$ ), with no evidence of heterogeneity ( $p=$ $0.101)$. When 2 studies $[26,34]$ were removed between SOX2 expression and clinical stage, the re-calculated OR remained significant $(\mathrm{OR}=3.43, p<0.001)$, with no heterogeneity $(p=0.234)$. When the study of $\mathrm{Ni}$ et al. [37] (2017) was deleted, the re-calculated result remained significant between SOX2 expression and $\mathrm{pT}$ stage $(\mathrm{OR}=$ $1.78, p<0.001)$, with no evidence of heterogeneity $(p=$ 0.458 ). When these 3 studies $[22,26,32]$ were deleted, the re-calculated result was still not associated with lymph node metastasis $(p=0.186)$, with no heterogeneity $(p=$ 0.141). When the study of Gupta et al. [39] (2018) was deleted, the re-calculated result was still not correlated with lymphovascular invasion ( $p=0.085)$, with no evidence of heterogeneity $(p=0.634)$.

Role of SOX2 in Breast Cancer
When 3 studies $[33,37,39]$ were removed, the re-calculated result was significantly changed, showing a negative association with ER status $(\mathrm{OR}=0.62, p=0.001)$ and no evidence of heterogeneity $(p=0.384)$. When the study of Abd El-Maqsoud et al. [33] (2014) was removed, the re-calculated result was still not correlated with PR status $(p=0.305)$, with no heterogeneity $(p=0.112)$. When 2 studies $[30,33]$ were removed, the re-calculated OR was still not associated with TN status $(p=0.644)$, resulting in no heterogeneity $(p=0.209)$.

\section{Publication Bias}

The results of Egger's test showed that slight publication bias was detected between SOX2 expression and tumor grade $(p=0.013)$ and TN status $(p=0.035)$ (online suppl. Fig. S1). But no publication bias was found between SOX2 expression and age, pT stage, lymph node metastasis, ER status, PR status, and HER2 status (all $p>$ 0.1) (online suppl. Fig. S1). 


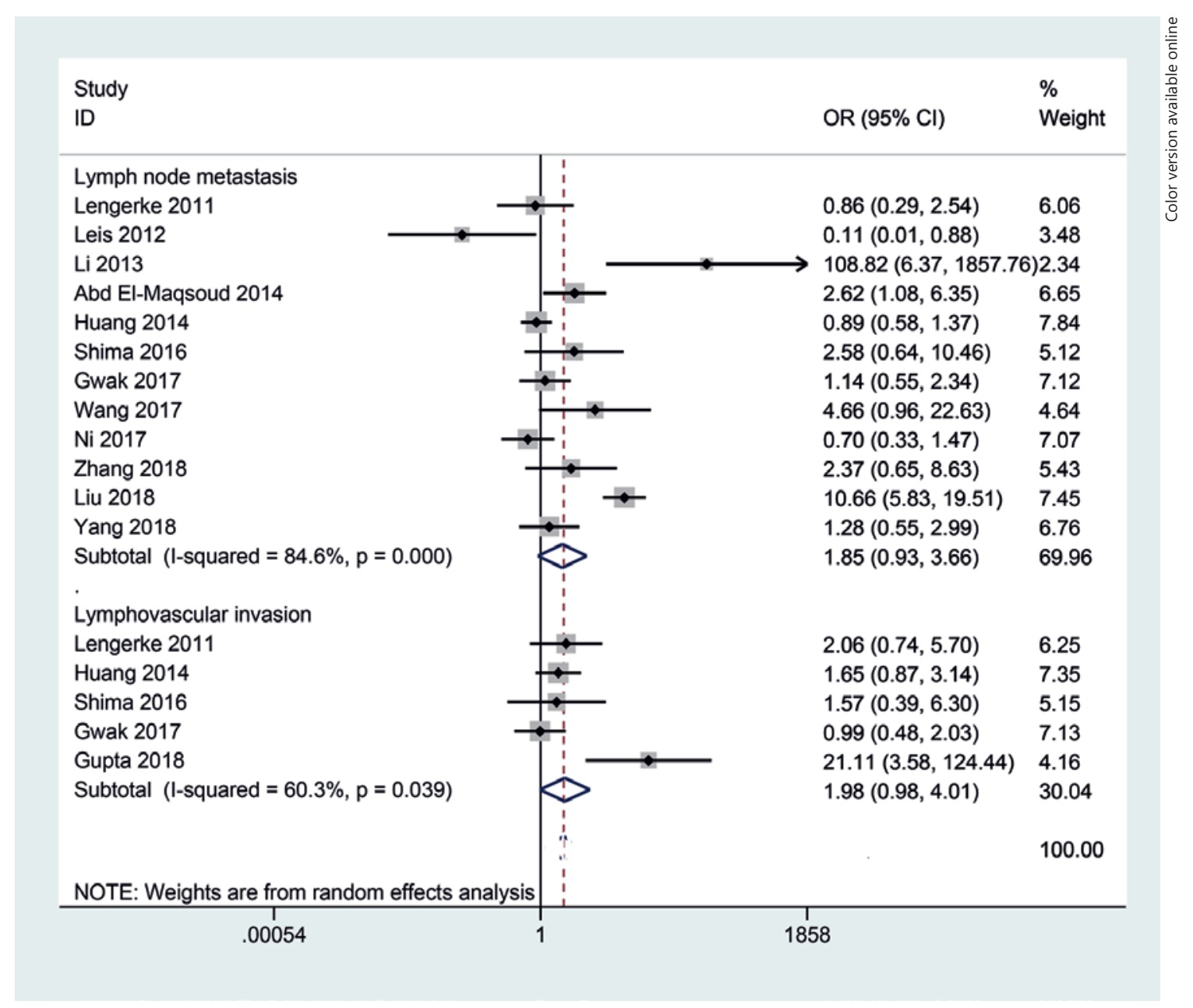

Fig. 5. Forest plot of the association between SOX2 expression and lymph node metastasis (positive vs. negative) and lymphovascular invasion (positive vs. negative).

\section{Discussion}

CSCs, a distinct population of cancer cells, are associated with tumor progression, metastasis, relapse, and resistance to conventional anticancer therapies $[42,43]$. Many CSC markers have been reported and may play important roles in the development, progression, prognosis, and resistance to therapy of human cancer such as CD133, ALDH1, podoplanin, and EpCAM [44-47]. SOX2 has been identified as an important CSC marker and plays critical roles in the maintenance of the stem cell state [14, 48]. It is correlated with the migration, invasion, and proliferation of cancer cells, and is also involved in resistance to treatment [14]. SOX2 expression is common across a wide range of human tumors, such as gastric, colorectal, lung, and ovarian cancers [16]. Its expression is closely related to worse prognosis in some types of human cancers $[17,18]$. SOX2 overexpression is found in cancer metastasis [49]. In recent years, numerous studies have reported that SOX2 is frequently expressed in patients with breast cancer $[20-23,40]$, and its expression may be as- sociated with an increase in tumor size, histological grade, lymph node metastasis, and high invasiveness of breast cancer [50]. However, the function of CSC marker SOX2 and its clinical role in patients with breast cancer remains significantly unclear. Based on 18 available studies with 3,080 patients with breast cancer, we conducted a systematic meta-analysis to analyze the clinical and molecular significance of SOX2 expression and its impact on the survival of breast cancer patients.

We analyzed whether SOX2 expression was correlated with the clinical and molecular features of patients with breast cancer. SOX2 expression was not associated with age factor $[20,22,23,26,33,35,37-39,41]$ and menopausal status $[22,30,33,34,36]$ among all studies. Our pooled results remained consistent, showing no correlation between SOX2 expression and age and menopausal status. No significant relationship was observed between SOX2 expression and lymph node metastasis and lymphovascular invasion of patients with breast cancer, which was consistent with the previous publications re- 


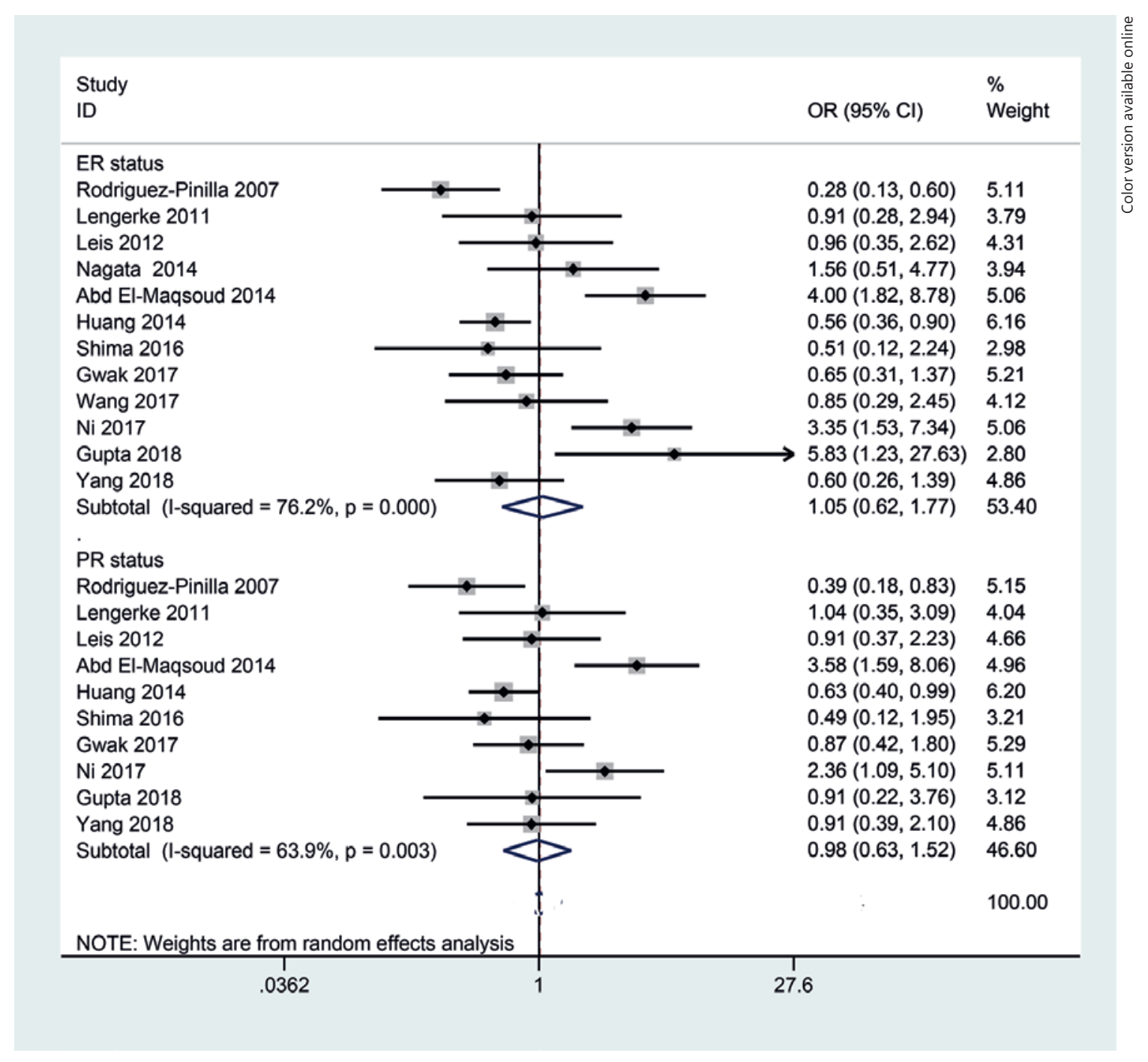

Fig. 6. Forest plot of the association between SOX2 expression and ER status (positive vs. negative) and PR status (positive vs. negative).

garding SOX2 expression with lymph node metastasis $[20,23,24,31,36,37,38,41]$ and lymphovascular invasion $[20,24,31,36]$. Some previous publications reported that SOX2 expression was positively linked to tumor grade $[5,20,21,23,24,32,33,36,38,39,41]$, clinical stage $[22,32,38]$, and pT stage $[20,23,30]$, which were consistent with our results that showed that SOX2 expression was associated with advanced tumor grade, clinical stage, and pT stage. This suggests that SOX2 expression plays an important role in the progression of breast cancer.

Our pooled data suggested that SOX2 expression was not correlated with ER status, PR status, and TN status of breast cancer, which were consistent with the previous studies regarding the correlation between SOX2 expression and ER status [23, 24, 26, 31, 34, 36, 38], PR status $[23,24,26,31,36,39]$, and TN status $[24,26,31,34,36$, 38]. Some studies reported that SOX2 expression was re- lated to HER2 status [26] and EGFR status [30]. Our results were consistent and showed that SOX2 expression was positively associated with HER2-positive and EGFRpositive patients with breast cancer.

We finally analyzed the prognostic effect of SOX2 expression on breast cancer patients using multivariate Cox survival analysis. We found that SOX2 expression was not related to OS, but was associated with worse DFS in breast cancer, suggesting that SOX2 may become a potential prognostic biomarker for predicting poor DFS of patients with breast cancer. More clinical studies with large sample sizes are necessary to further confirm the prognostic value of SOX2 expression in patients with breast cancer.

There were some limitations in this meta-analysis. First, although we performed sensitivity analyses to estimate the possible sources of heterogeneity and the stability of the re-calculated results, the potential sources of 


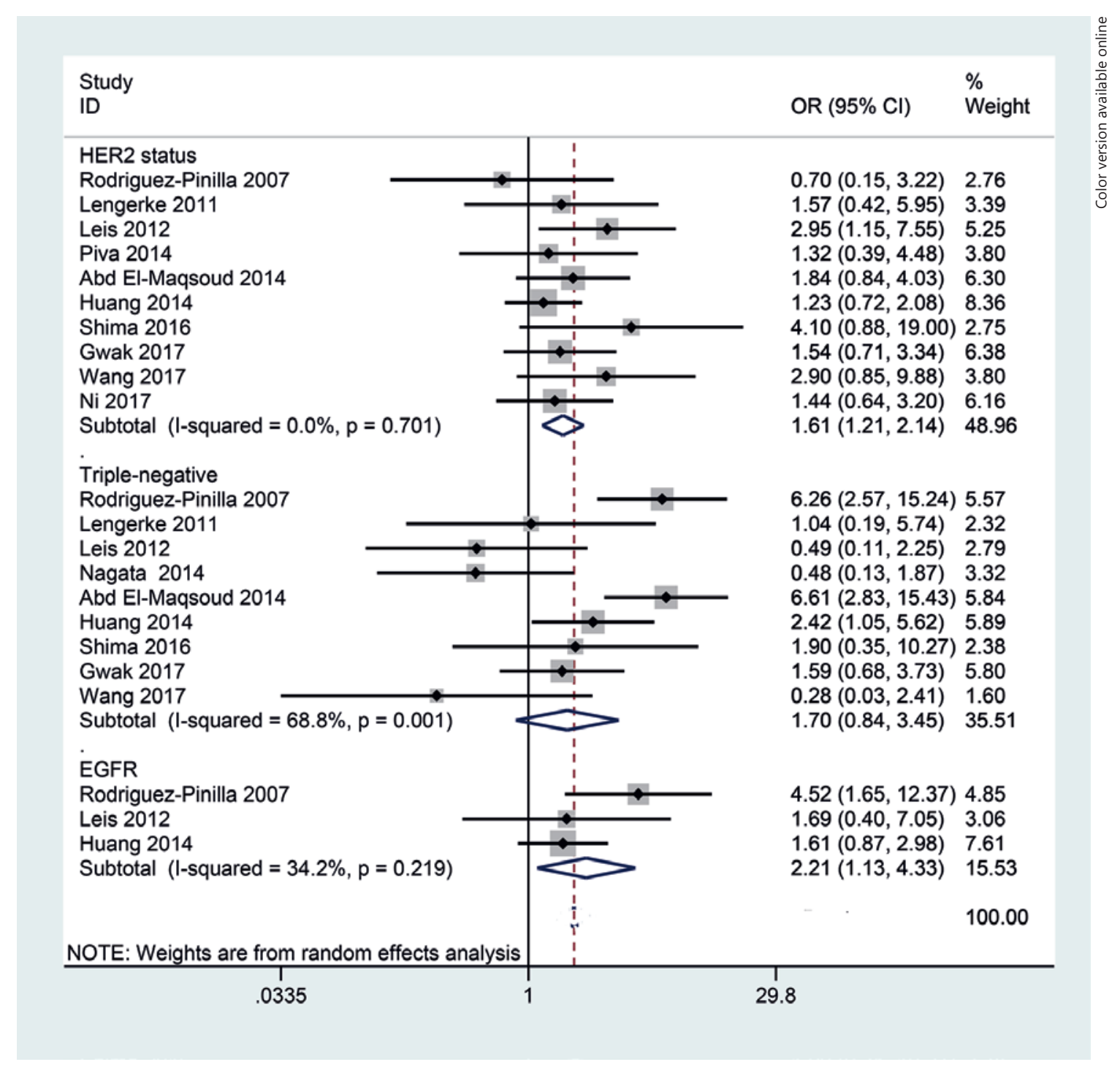

Fig. 7. Forest plot of the association between SOX2 expression and HER2 status (positive vs. negative), triplenegative status (yes vs. no), and EGFR status (positive vs. negative).

heterogeneity were not clearly found. The potential reasons were as follows: (1) the sources of many SOX2 antibodies were different; (2) the cut-off values of SOX2 expression were defined as "positive" or "negative" from the original articles, which were different in many eligible studies; and (3) although 11 studies of the total 18 studies showed cell nuclear distribution, cell distribution of the SOX2 protein was different, the remaining 5 studies were not clear, and only 2 studies showed cell membrane or cytoplasm/nuclear. These reasons may cause unavoidable heterogeneity. Thus, the determination of SOX2 expression as "positivity" and the uniform use of SOX2 antibodies should be conducted based on a uniform standard, which should be done in the future. The SOX2 protein was examined for use in cell nuclear staining of specimens, which should be recommended in the future. Additionally, sensitivity analysis suggested that the result be- tween SOX2 expression and ER status was not stable; more studies are needed to validate this result in the future. Second, slight publication bias was measured between SOX2 expression and tumor grade and TN status. Although a number of databases were completely searched to minimize the possible publication bias, the included studies were enrolled from articles published in English. Publications of other styles, such as articles published in other languages and conference abstracts, were lacking due to insufficient information and data. Moreover, papers with negative results are more difficult to be accepted and published than articles with positive results. These reasons may lead to potential bias. Third, additional clinical studies with large study populations using multivariate Cox survival analysis are essential to further confirm the prognostic significance of SOX2 expression in patients with breast cancer. 


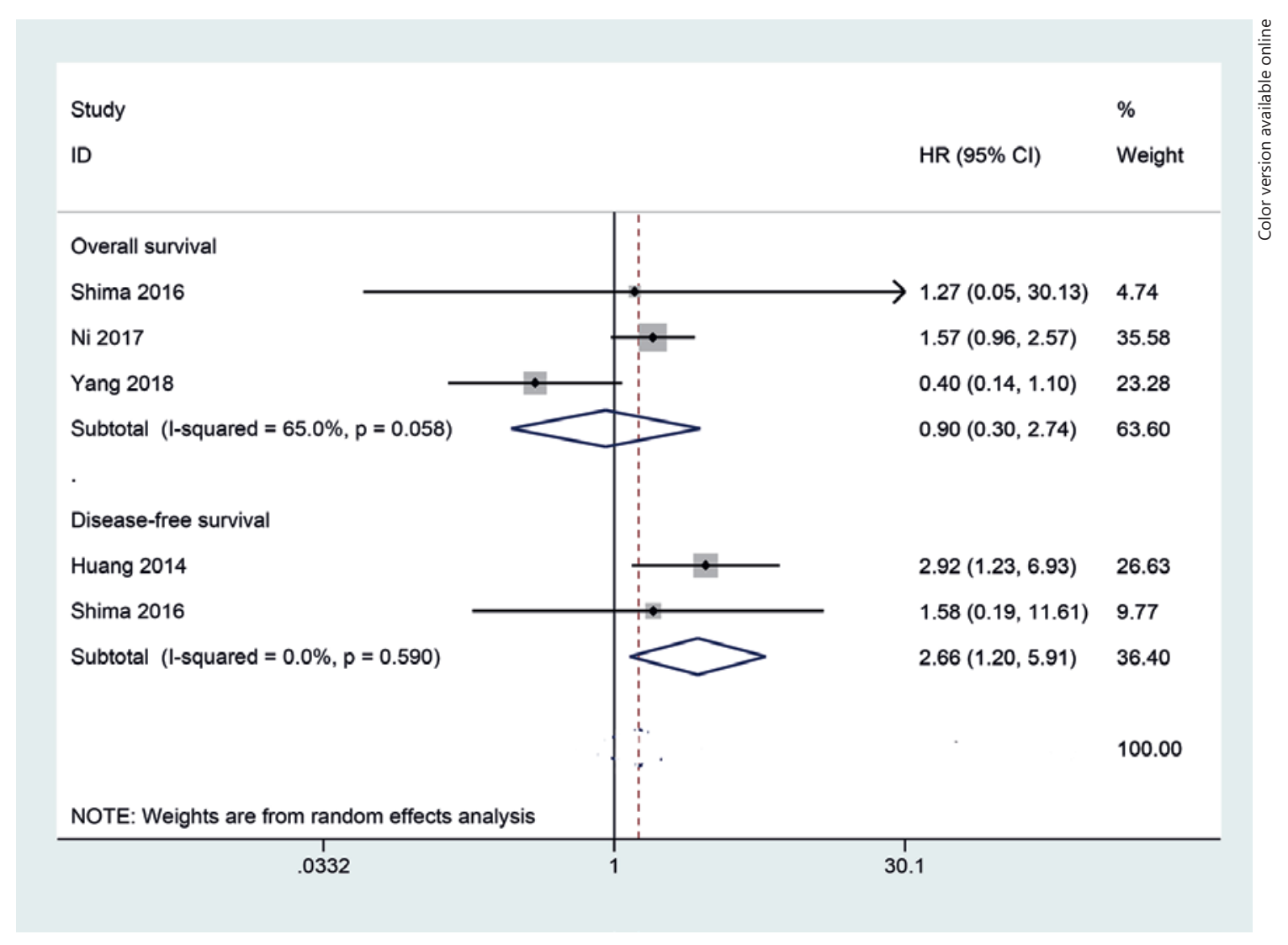

Fig. 8. Forest plot of the association between SOX2 expression and the prognosis using multivariate Cox survival analysis in overall survival and disease-free survival.

In summary, this meta-analysis showed that SOX2 expression was not associated with age, menopausal status, lymph node metastasis, lymphovascular invasion, ER status, PR status, TN status, and OS, but was closely correlated with advanced tumor grade, clinical stage, $\mathrm{pT}$ stage, HER2 positive status, EGFR positive status, and worse DFS of patients with breast cancer. These data suggest that SOX2 expression is associated with breast cancer progression, HER2 status, and EGFR status, and may become a promising prognostic biomarker for the prediction of worse DFS. More large-scale prospective clinical studies are required to further validate the prognostic effect of SOX2 expression in breast cancer.

\section{Statement of Ethics}

This meta-analysis was conducted according to the PRISMA statement criteria.

\section{Disclosure Statement}

The authors declare that they have no conflict of interest.

\section{Author Contributions}

G.Z., X.W., and Z.L. contributed to the study conception and design. G.Z. and X.W. drafted the article. All authors contributed to the analyses and interpretation of the data and completion of figures and tables. All authors read and approved the final manuscript.

\section{References}

1 Bray F, Ferlay J, Soerjomataram I, Siegel RL, Torre LA, Jemal A. Global cancer statistics 2018: GLOBOCAN estimates of incidence and mortality worldwide for 36 cancers in 185 countries. CA Cancer J Clin. 2018 Nov;68(6):394-424.

2 Dey N, Barwick BG, Moreno CS, OrdanicKodani M, Chen Z, Oprea-Ilies G, et al. Wnt signaling in triple negative breast cancer is associated with metastasis. BMC Cancer. 2013 Nov; 13(1):537.

3 Van Poznak C, Somerfield MR, Bast RC, Cristofanilli M, Goetz MP, Gonzalez-Angulo AM, et al. Use of Biomarkers to Guide Decisions on Systemic Therapy for Women With Metastatic Breast Cancer: American Society of Clinical Oncology Clinical Practice Guideline. J Clin Oncol. 2015 Aug;33(24):2695-704.

4 Berry DA, Cronin KA, Plevritis SK, Fryback DG, Clarke L, Zelen M, et al.; Cancer Intervention and Surveillance Modeling Network (CISNET) Collaborators. Effect of screening and adjuvant therapy on mortality from breast cancer. N Engl J Med. 2005 Oct;353(17):1784-92. 
5 Matikas A, Foukakis T, Bergh J. Tackling endocrine resistance in ER-positive HER2-negative advanced breast cancer: A tale of imprecision medicine. Crit Rev Oncol Hematol. 2017 Jun;114:91-101.

6 Lei Y, Zhang D, Yu J, Dong H, Zhang J, Yang S. Targeting autophagy in cancer stem cells as an anticancer therapy. Cancer Lett. 2017 May; 393:33-9.

7 Han S, Huang T, Wu X, Wang X, Liu S, Yang W, et al. Prognostic value of CD133 and SOX2 in advanced cancer. J Oncol. 2019 Jan;2019: 3905817.

8 Eun K, Ham SW, Kim H. Cancer stem cell heterogeneity: origin and new perspectives on CSC targeting. BMB Rep. 2017 Mar;50(3): 117-25.

9 Han S, Huang T, Li W, Wang X, Wu X, Liu S, et al. Prognostic value of CD44 and its isoforms in advanced cancer: a systematic metaanalysis with trial sequential analysis. Front Oncol. 2019 Feb;9:39.

10 Liskova A, Kubatka P, Samec M, Zubor P, Mlyncek M, Bielik T, et al. Dietary Phytochemicals Targeting Cancer Stem Cells. Molecules. 2019 Mar;24(5):E899.

11 Annovazzi L, Mellai M, Caldera V, Valente G, Schiffer D. SOX2 expression and amplification in gliomas and glioma cell lines. Cancer Genomics Proteomics. 2011 May-Jun;8(3): 139-47.

12 Koike T, Wakabayashi T, Mori T, Takamori Y, Hirahara Y, Yamada H. Sox2 in the adult rat sensory nervous system. Histochem Cell Biol. 2014 Mar;141(3):301-9.

13 Mansouri S, Nejad R, Karabork M, Ekinci C, Solaroglu I, Aldape KD, et al. Sox2: regulation of expression and contribution to brain tumors. CNS Oncol. 2016 Jul;5(3):159-73.

14 Hüser L, Novak D, Umansky V, Altevogt P, Utikal J. Targeting SOX2 in anticancer therapy. Expert Opin Ther Targets. 2018 Dec; 22(12):983-91.

15 Carrasco-Garcia E, Santos JC, Garcia I, Brianti M, García-Puga M, Pedrazzoli J Jr, et al. Paradoxical role of SOX2 in gastric cancer. Am J Cancer Res. 2016 Mar;6(4):701-13.

16 Wuebben EL, Rizzino A. The dark side of SOX2: cancer - a comprehensive overview. Oncotarget. 2017 Jul;8(27):44917-43.

17 Li W, Li B, Wang R, Huang D, Jin W, Yang S SOX2 as prognostic factor in head and neck cancer: a systematic review and meta-analysis. Acta Otolaryngol. 2014 Nov; 134(11) 1101-8.

18 Du XM, Wang LH, Chen XW, Li YX, Li YC, Cao YW. Prognostic value of Sox2 expression in digestive tract cancers: A meta-analysis. J Huazhong Univ Sci Technolog Med Sci. 2016 Jun;36(3):305-12.

19 Shao W, Chen H, He J. The role of SOX-2 on the survival of patients with non-small cell lung cancer. J Thorac Dis. 2015 Jul;7(7):11138.

20 Huang YH, Luo MH, Ni YB, Tsang JY, Chan SK, Lui PC, et al. Increased SOX2 expression in less differentiated breast carcinomas and their lymph node metastases. Histopathology. 2014 Mar;64(4):494-503.

21 Kündig P, Giesen C, Jackson H, Bodenmiller B, Papassotirolopus B, Freiberger SN, et al. Limited utility of tissue micro-arrays in de- tecting intra-tumoral heterogeneity in stem cell characteristics and tumor progression markers in breast cancer. J Transl Med. 2018 May;16(1):118.

22 Liu P, Tang H, Song C, Wang J, Chen B, Huang X, et al. SOX2 promotes cell proliferation and metastasis in triple negative breast cancer. Front Pharmacol. 2018 Aug;9:942.

23 Yang F, Zhang J, Yang H. OCT4, SOX2, and NANOG positive expression correlates with poor differentiation, advanced disease stages, and worse overall survival in HER2+ breast cancer patients. Onco Targets Ther. 2018 Nov; 11:7873-81.

24 Gwak JM, Kim M, Kim HJ, Jang MH, Park SY. Expression of embryonal stem cell transcription factors in breast cancer: Oct 4 as an indicator for poor clinical outcome and tamoxifen resistance. Oncotarget. 2017 May; 8(22): 36305-18.

25 Liberati A, Altman DG, Tetzlaff J, Mulrow C, Gøtzsche PC, Ioannidis JP, et al. The PRISMA statement for reporting systematic reviews and meta-analyses of studies that evaluate healthcare interventions: explanation and elaboration. BMJ. 2009 Jul;339 jul21 1:b2700.

26 Leis O, Eguiara A, Lopez-Arribillaga E, Alberdi MJ, Hernandez-Garcia S, Elorriaga K, et al. Sox 2 expression in breast tumours and activation in breast cancer stem cells. Oncogene. 2012 Mar;31(11):1354-65.

27 Zintzaras E, Ioannidis JP. HEGESMA: genome search meta-analysis and heterogeneity testing. Bioinformatics. 2005 Sep;21(18): 3672-3.

28 Lau J, Ioannidis JP, Schmid CH. Quantitative synthesis in systematic reviews. Ann Intern Med. 1997 Nov;127(9):820-6.

29 Egger M, Davey Smith G, Schneider M, Minder C. Bias in meta-analysis detected by a simple, graphical test. BMJ. 1997 Sep;315(7109): 629-34.

30 Rodriguez-Pinilla SM, Sarrio D, MorenoBueno G, Rodriguez-Gil Y, Martinez MA, Hernandez L, et al. Sox2: a possible driver of the basal-like phenotype in sporadic breast cancer. Mod Pathol. 2007 Apr;20(4):474-81.

31 Lengerke C, Fehm T, Kurth R, Neubauer H, Scheble V, Müller F, et al. Expression of the embryonic stem cell marker SOX2 in earlystage breast carcinoma. BMC Cancer. 2011 Jan;11(1):42.

32 Li X, Xu Y, Chen Y, Chen S, Jia X, Sun T, et al. SOX2 promotes tumor metastasis by stimulating epithelial-to-mesenchymal transition via regulation of $\mathrm{WNT} / \beta$-catenin signal network. Cancer Lett. 2013 Aug;336(2): 379-89.

33 Abd El-Maqsoud NM, Abd El-Rehim DM. Clinicopathologic implications of EpCAM and Sox2 expression in breast cancer. Clin Breast Cancer. 2014 Feb;14(1):e1-9.

34 Nagata T, Shimada Y, Sekine S, Hori R, Matsui K, Okumura T, et al. Prognostic significance of NANOG and KLF4 for breast cancer. Breast Cancer. 2014 Jan;21(1):96-101.

35 Piva M, Domenici G, Iriondo O, Rábano M, Simões BM, Comaills V, et al. Sox 2 promotes tamoxifen resistance in breast cancer cells. EMBO Mol Med. 2014 Jan;6(1):66-79.

36 Shima H, Kutomi G, Satomi F, Maeda H, Hirohashi Y, Hasegawa T, et al. SOX2 and
ALDH1 as Predictors of Operable Breast Cancer. Anticancer Res. 2016 Jun;36(6):2945-53.

37 Ni W, Yang Z, Qi W, Cui C, Cui Y, Xuan Y. Glil is a potential cancer stem cell marker and predicts poor prognosis in ductal breast carcinoma. Hum Pathol. 2017 Nov;69:38-45.

38 Wang J, Zeng H, Li H, Chen T, Wang L, Zhang $\mathrm{K}$, et al. MicroRNA-101 Inhibits Growth, Proliferation and Migration and Induces Apoptosis of Breast Cancer Cells by Targeting Sex-Determining Region Y-Box 2. Cell Physiol Biochem. 2017;43(2):717-32.

39 Gupta N, Gopal K, Wu C, Alshareef A, Chow A, Wu F, et al. Phosphorylation of Sox2 at Threonine 116 is a Potential Marker to Identify a Subset of Breast Cancer Cells with High Tumorigenecity and Stem-Like Features. Cancers (Basel). 2018 Feb;10(2):E41.

40 Li C, Fan Q, Quan H, Nie M, Luo Y, Wang L. The three branches of the unfolded protein response exhibit differential significance in breast cancer growth and stemness. Exp Cell Res. 2018 Jun;367(2):170-85.

41 Zhang JM, Wei K, Jiang M. OCT4 but not SOX2 expression correlates with worse prognosis in surgical patients with triple-negative breast cancer. Breast Cancer. 2018 Jul;25(4): 447-55.

42 Crupi MJ, Bell JC, Singaravelu R. Concise Review: Targeting Cancer Stem Cells and Their Supporting Niche Using Oncolytic Viruses. Stem Cells. 2019 Jun;37(6):716-23.

43 Han S, Huang T, Wu X, Wang X, Li W, Liu $S$, et al. Prognostic value of ALDH1 and Nestin in advanced cancer: a systematic meta-analysis with trial sequential analysis. Ther Adv Med Oncol. 2019 Feb;11: 1758835919830831.

44 Han S, Zong S, Shi Q, Li H, Liu S, Yang W, et al. Is Ep-CAM expression a diagnostic and prognostic biomarker for colorectal cancer? A systematic meta-analysis. EBioMedicine. 2017 Jun;20:61-9.

45 Panaccione A, Guo Y, Yarbrough WG, Ivanov SV. Expression profiling of clinical specimens supports the existence of neural progenitor-like stem cells in basal breast cancers. Clin Breast Cancer. 2017 Jul;17(4):298-306. e7.

46 Kazama S, Kishikawa J, Kiyomatsu T, Kawai K, Nozawa H, Ishihara S, et al. Expression of the stem cell marker CD133 is related to tumor development in colorectal carcinogenesis. Asian J Surg. 2018 May;41(3):274-8.

47 Saluja TS, Ali M, Mishra P, Kumar V, Singh SK. Prognostic Value of Cancer Stem Cell Markers in Potentially Malignant Disorders of Oral Mucosa: A Meta-analysis. Cancer Epidemiol Biomarkers Prev. 2019 Jan;28(1): 144-53.

48 Zhu F, Qian W, Zhang H, Liang Y, Wu M, Zhang Y, et al. SOX2 Is a Marker for Stem-like Tumor Cells in Bladder Cancer. Stem Cell Reports. 2017 Aug;9(2):429-37.

49 Javaeed A, Ghauri SK. Metastatic potential and prognostic significance of SOX2: A metaanalysis. World J Clin Oncol. 2019 Jun;10(6): 234-46.

50 Feng X, Lu M. Expression of sex-determining region Y-box protein 2 in breast cancer and its clinical significance. Saudi Med J. 2017 Jul; 38(7):685-90. 